\title{
Surface Interaction Forces of Cellulose Nanocrystals Grafted with Thermo-responsive Polymer Brushes
}

\begin{tabular}{|c|c|}
\hline Journal: & Biomacromolecules \\
\hline Manuscript ID: & bm-2011-00551p.R1 \\
\hline Manuscript Type: & Article \\
\hline $\begin{array}{r}\text { Date Submitted by the } \\
\text { Author: }\end{array}$ & $\mathrm{n} / \mathrm{a}$ \\
\hline Complete List of Authors: & $\begin{array}{l}\text { Zoppe, Justin; North Carolina State University, Forest Biomaterials } \\
\text { Österberg, Monika; Aalto University, Forest Products Technology } \\
\text { Venditti, Richard; NCSU, Wood and paper Science } \\
\text { Laine, Janne; Aalto University, Forest Products Technology } \\
\text { Rojas, Orlando; North Carolina State University, Department of } \\
\text { Forest Biomaterials }\end{array}$ \\
\hline
\end{tabular}

\section{SCHOLARONE ${ }^{\text {W }}$ \\ Manuscripts}




\title{
Surface Interaction Forces of Cellulose Nanocrystals
}

\section{Grafted with Thermo-responsive Polymer Brushes}

\author{
Justin O. Zoppe,${ }^{\S}$ Monika Österberg, ${ }^{\dagger *}$ Richard A. Venditti, ${ }^{\S}$ Janne Laine ${ }^{\dagger}$ and \\ Orlando J. Rojas, ${ }^{\circledR * *}$ \\ ${ }^{\S}$ Department of Forest Biomaterials, North Carolina State University, Campus Box 8005, Raleigh, \\ NC USA \\ ${ }^{\dagger}$ Department of Forest Products Technology, School of Science and Technology, Aalto University, \\ P.O. Box 16300, Aalto FIN-00076 Finland
}

*Corresponding author email addresses: ojrojas@ncsu.edu, monika.osterberg@aalto.fi

\begin{abstract}
The colloidal stability and thermo-responsive behavior of poly( $\mathrm{N}$-isopropylacrylamide) brushes grafted from cellulose nanocrystals (CNCs) of varying graft densities and molecular weights was investigated. Indication of the grafted polymer brushes was obtained after AFM imaging of CNCs adsorbed on silica. Also, aggregation of the nanoparticles carrying grafts of high degree of polymerization was observed. The responsiveness of grafted CNCs in aqueous dispersions and as an ultrathin film was evaluated by using light scattering, viscosimetry and Colloidal Probe Microscopy (CPM). Light transmittance measurements showed temperature-dependent aggregation originating from the different graft densities and molecular weights. The lower critical solution temperature (LCST) of grafted poly(NiPAAm) brushes was found to decrease with the ionic strength as is the
\end{abstract}




\section{Introduction}

Understanding the interfacial forces between particles with adsorbed polymer layers has great relevance, not only in the area of colloid and surface chemistry, but also in the design of new materials in an array of emerging technologies. ${ }^{1}$ Coating, papermaking, water treatment, emulsification, oil and mineral extraction, are among the many applications that are dependent on such interfacial interactions. ${ }^{2}$ The control of the adhesion, lubrication and rheological behaviors as well as colloidal stability implicit in these systems can be gained by physical adsorption and covalent grafting of polymers on surfaces. ${ }^{2-4}$ Within the development of biomedical and smart devices lies more recent interests in the manipulation of bio-interfaces for drug-delivery, biosensing, and cell adhesion, etc. $^{5-8}$

Stimuli-responsive polymers have been extensively studied, mostly driven by new applications in biotechnology. ${ }^{9}$ Since the first reported synthesis of N-isopropylacrylamide (NiPAAm) in $1956,{ }^{10}$ poly(N-isopropylacrylamide) (poly(NiPAAm)) has been one of the most widely studied thermo- 
responsive polymers due to its coil-to-globule transition at $32{ }^{\circ} \mathrm{C}$ in aqueous solution, also called the Lower Critical Solution Temperature (LCST). ${ }^{11}$ This transition, which occurs within the proximity of physiological temperature, comes about from thermal dehydrations of the polymer chain that yields an entropic gain in free energy of the system. The LCST of poly(NiPAAm) has been known for decades, ${ }^{12}$ and has been most recently explained by Matsuyama et al. ${ }^{13}$ as the mixing entropy produced by solvation. Importantly, factors such as ionic strength and $\mathrm{pH}$ of the respective aqueous dispersion or solution can affect the LCST. ${ }^{14-16}$

Great interest in poly(NiPAAm) grafts has developed due to the ability of such layers to reversibly attach and detach cultured cells above and below the LCST, respectively. ${ }^{17}$ Among the approaches to generate polymer brushes based on poly(NiPAAm), "grafting onto" and "grafting from" methods are the most prevalent ones. ${ }^{14,}$ 18-27 The first report on the thermo-responsive behavior of poly(NiPAAm) grafts was performed by Okahata et al., ${ }^{28}$ in which the permeability of $\mathrm{NaCl}$ and dyes through a nylon capsule membrane grafted with poly(NiPAAm) could be reversibly controlled by solution temperature. Despite its importance, the characterization of the surface phase transition of thermo-responsive polymer coatings has proven to be challenging, ${ }^{29}$ however, a number of efforts have been undertaken by using contact angle, AFM, colloidal probe microscopy (CPM), quartz crystal microgravimetry, etc. ${ }^{14,18,20,27,30-34}$

Important contributions relevant to surface responsiveness of grafted polymer brushes are the type and magnitude of interaction forces involved when grafted surfaces are brought into close proximity. In addition to DLVO van der Waals and electrostatic interactions, non-specific interactions, specifically steric repulsion and polymer bridging, are expected to play an important role. Some of the theories developed to describe steric forces of tethered or adsorbed polymers at interfaces include those from Alexander ${ }^{35}$-de Gennes $^{36}$ and Milner-Witten-Cates (MWC) ${ }^{37}$, which differ in the description of the segment density profile of the adsorbed or grafted polymer layer.

In addition to well established systems in which polymer grafting has made important inroads, applications involving lignocellulosics have emerged in recent years. Examples are provided by reported efforts to develop "smart" materials based on cellulosic materials 38,39 including filter 
paper, ${ }^{19}$ cellulose nanocrystals (CNCs) or whiskers, ${ }^{40-42}$ and nanofibrillated cellulose. ${ }^{43}$ CNCs in particular, are attractive due to their unique surface, optical and mechanical properties. ${ }^{44,45}$

Interfacial forces between cellulosic surfaces have been previously studied using the colloidal probe technique ${ }^{46-62}$ and the Surface Force Apparatus. ${ }^{63-65}$ Lefebvre et al. ${ }^{66}$ first studied deflection versus distance curves of standard silicon nitride tips on spin-coated cellulose nanocrystal films and adsorbed layers of poly(diallyldimethylammonium chloride) via AFM. The surface forces between a mostly amorphous colloidal cellulose sphere and cellulose surfaces of varying crystallinity, one being a film of sulfate-stabilized CNCs has been studied recently. ${ }^{49}$ The observed forces corresponded well to DLVO behaviors due to dominant electrostatic forces of the sulfate-charged nanoparticles. In addition, amorphous cellulose films resulted in surface forces of predominantly steric origin. Cranston et al. ${ }^{62}$ have reported on direct surface force measurements of polyelectrolyte multilayer films containing CNCs and poly(allylamine hydrochloride) (PAH) with varying ionic strength and pH. In recent work the surface interactions of polyelectrolyte-cellulose systems were investigated utilizing colloidal probe AFM with cellulosic spheres in which the measured surface forces correlated well with practical observations related to flocculation. ${ }^{51}$

In our previous publication, ${ }^{42}$ we reported on the synthesis of poly(NiPAAm) brushes grafted from CNCs via surface-initiated single-electron transfer living radical polymerization (SI-SET-LRP). The resulting materials were proposed to not only provide steric stabilization of colloidal dispersions of bio-based CNCs, but also to enable nanotechnological systems that may benefit from thermoresponsive functionalities. The aim of the current study was therefore to probe the thermoresponsive behavior of poly(NiPAAm) brushes grafted from CNCs (poly(NiPAAm)-g-CNCs) in aqueous dispersion and as ultrathin films. As such, unmodified and grafted CNCs were probed and compared using viscometry, light transmittance, and colloidal probe microscopy (CPM).

\section{Experimental}

Materials. Pure ramie fibers were obtained from Stucken Melchers GmbH \& Co. (Germany). 2bromoisobutyryl bromide (BriB), 2-dimethylaminopyridine (DMAP), tetrahydrofuran (THF, 99\%), N-isopropylacryalamide (NiPAAm), copper (I) bromide and $N, N, N^{\prime}, N^{\prime \prime}, N^{\prime \prime}-$ pentamethyldiethylenetriamine (PMDETA), were all obtained from Sigma-Aldrich. Sulfuric acid 
(95\%), acetic acid (glacial), triethylamine (TEA, 99.5\%), acetone (99\%), ethanol (95\%), methanol (99\%), sodium hydroxide pellets, and sodium chlorite were all purchased from Fisher Scientific. All solvents were dried over molecular sieves ( $3 \AA$, 4-8 mesh beads, Sigma-Aldrich) for $48 \mathrm{~h}$ before use.

Preparation of cellulose nanocrystals (CNCS), poly(NiPAAm)-g-CNCs and respective ultrathin films. Details about the procedure employed to prepare $\mathrm{CNCs}$ and $\mathrm{CNCs}$ grafted with poly(NiPAAm) (poly(NiPAAm)-g-CNCs) can be found in our earlier publication. ${ }^{42}$ In order to prepare ultrathin films of $\mathrm{CNC}$ and poly(NiPAAm)-g-CNCs, the respective aqueous dispersion was diluted to the desired concentration and spin coated (4000 rpm for ca. 30 seconds) onto UV-treated silicon wafers. In the case of unmodified CNCs an anchoring layer of polyethyleneimine was preadsorbed on the wafers to increase adhesion of the sulfate-charged CNCs. Poly(NiPAAm)-g-CNCs were spin-coated directly without the need of anchoring polymer due to the presence of secondary amine moieties. The surfaces were then dried either at room temperature overnight or at $80{ }^{\circ} \mathrm{C}$ for 1 hour, before further experiments.

Light scattering. Aqueous dispersions of CNCs $(0.088 \mathrm{wt} \%)$ contained in a $250 \mathrm{~mL}$ Erlenmeyer flask were first subjected to sonication using an ultrasonic microtip (Branson Sonifier S-450 D, Danbury, USA). The dispersions were then pumped constantly at a rate of $300 \mathrm{~mL} / \mathrm{min}$ through a light scattering apparatus (Turbiscan Online by Formulaction, France). The temperature was first equilibrated at $20{ }^{\circ} \mathrm{C}$, followed by the placement of the flask into a water bath set at $50{ }^{\circ} \mathrm{C}$. The temperature of the aqueous dispersion and light transmittance (detection angle $0^{\circ}$ ) was recorded every second over a 20 minute time period, while the system was heated. The LCST of free and grafted poly(NiPAAm) was taken as the onset of decreased $\%$ light transmittance versus temperature.

Rheology. Viscosity measurements of aqueous dispersions of CNCs and poly(NiPAAm)-g-CNCs were carried out using a TA Instruments AR 2000 Rheometer. A Peltier plate and $25 \mathrm{~mm} 1.59^{\circ}$ cone geometry was used at a constant shear rate of $150 \mathrm{~s}^{-1}$. Viscosity was monitored at a temperature ramp range of 25 to $40{ }^{\circ} \mathrm{C}$.

Atomic Force Microscopy (AFM). Ultrathin films of CNCs and poly(NiPAAm)-g-CNCs were imaged using a scanning probe microscope (model Multimode Nanoscope IIIa, Digital Instruments) 
in tapping mode, which was equipped with an $\mathrm{E}$ or $\mathrm{J}$ scanner and with noncoated silicon-etched probes (model LTESP, Veeco).

Surface Force Measurements with the Colloidal Probe Technique. Force measurements of CNCs and poly(NiPAAm)-g-CNCs films were performed in liquid media using a scanning probe microscope (model Multimode Nanoscope IIIa, Digital Instruments) in contact mode. The microscope was equipped with a $\mathrm{J}$ scanner and triangular tipless silicon nitride probes with spring constants between $0.1-0.2 \mathrm{~N} / \mathrm{m}$ (model NP-O10, Veeco) calculated by the thermal noise method. ${ }^{67} \mathrm{~A}$ 25-45 $\mu \mathrm{m}$ silica sphere was attached to the tipless silicon nitride probes according to the method of Ducker et al. ${ }^{68,69}$ Each probe was UV-treated and cleaned thoroughly with water:ethanol solution immediately before use. The forces were normalized with the radius of each silica sphere. Scanning Probe Image Processor software (SPIP, Image Metrology, Denmark) was used to process force curves and calculate pull-off forces. The separation distances obtained were taken as relative to a hard wall contact.

\section{Results and Discussion}

CNCs from ramie fibers were produced and characterized according to a procedure reported previosly. ${ }^{42}$ The obtained CNCs were determined to have transverse dimensions of 3-15 nm and 50$250 \mathrm{~nm}$ in length. Poly(N-isopropylacrylamide) was grafted from CNCs via surface-initiated singleelectron transfer living radical polymerization (SI-SET-LRP). The grafting density was varied by using different amounts of the 2-bromoisobutyryl bromide initiator (BriB) with respect to the total hydroxyl groups in cellulose and denoted here as the molar ratio between initiator [Br] and anhydroglucose units $[\mathrm{AGU}]$ in $\mathrm{CNCs}([\mathrm{Br}]:[\mathrm{AGU}]): 5: 3,5: 4,5: 6$, and 5:12. For simplicity, these are respectively referred to as very high (VHD), high (HD), medium (MD), and low (LD) density poly(NiPAAm)-grafted CNCs (poly(NiPAAm)-g-CNCs). We note that only the glucose units on the surface of the nanoparticle were accessible to grafting. Reporting the fraction of surface accessible glucose units that were grafted would have been appropriate since this would certainly consider the limiting number of positions where grafting can occur and increase the reported graft density. 
To vary the chain length of the grafted polymer chains a series of poly(NiPAAm)-g-CNCs were prepared by varying the ratio between the immobilized initiator and the NiPAAm monomer at three ratios of monomer to total anhydroglucose units ([NiPAAm]:[AGU]), namely, 50:3, 75:3, and 100:3. In this series the graft density was kept constant at $[\mathrm{Br}]:[\mathrm{AGU}]=5: 4$ (MD condition). The grafted CNCs that were obtained are thereafter referred to as low (LDP), medium (MDP), and high (HDP) degree of polymerization, respectively. Since several poly (NiPAAm) chains were grafted from a single CNC particle the chains could behave in a cooperative manner and could perhaps be described in terms of hyperbranched and dendritic systems. However, no attempt was made to study in detail such structures; instead, the experimental values of molecular weight and polydispersity index used here follow our previous work. ${ }^{42}$

Thin films of cellulose nanocrystals were produced via spin-coating onto silicon wafers and were dried at room temperature overnight, as indicated in the Methods section. AFM images of the obtained films are shown in Figure 1. The images of LD poly(NiPAAm)-g-CNCs showed similar uniformity to unmodified CNCs, however the grafted crystals were slightly broader and distorted in the edges, as seen in Figure 1. In general, uniform surface coverage over the solid support was observed in films obtained from unmodified CNCs. The uniformity of the films obtained varied greatly with various graft densities and degree of polymerization. In general, more extensive grafting from the surfaces of CNCs produced larger aggregates during the spin-coating process. Similar observations were made in the case of CNC films that where dried at $80{ }^{\circ} \mathrm{C}$ after spin coating. While the effects of drying and dewetting on surface aggregation cannot be discounted, the evidence seemed to indicate that $\mathrm{CNC}$ aggregates occurred in the precursor aqueous dispersions and were deposited as such (as will be discussed later with light transmittance experiments). Significantly, CNC aggregation in the films was favored in the case of polymer grafts of larger density and degree of polymerization.

The distortion around the grafted CNCs increased consistently with increasing grafting density and Figure 1(f) of HDP poly(NiPAAm)-g-CNCs shows without uncertainty a polymer "halo" surrounding the nanoparticles that is even more pronounced in the respective phase image, Figure 1(f, inset). This may be taken as indication of the presence of a soft polymer layer around the CNCs. 
MD grafted CNCs showed a similar trend in uniformity, although small aggregates were more prominent. In the case of HD grafted CNCs, spin-coating was unsuccessful most likely due to very large aggregates formed in dispersion which could not adhere to the oxidized silicon wafer surface. VHD grafted CNCs gave films with a distribution of small aggregates as shown in Figure 1(e). Overall, it was observed that the degree of polymerization had a pronounced effect on the aggregate sizes of spin-coated films. MDP and HDP grafted CNCs yielded aggregates a few microns in lateral dimension, most likely due to the screening of electrostatic forces of sulfate groups by longer poly(NiPAAm) chains.
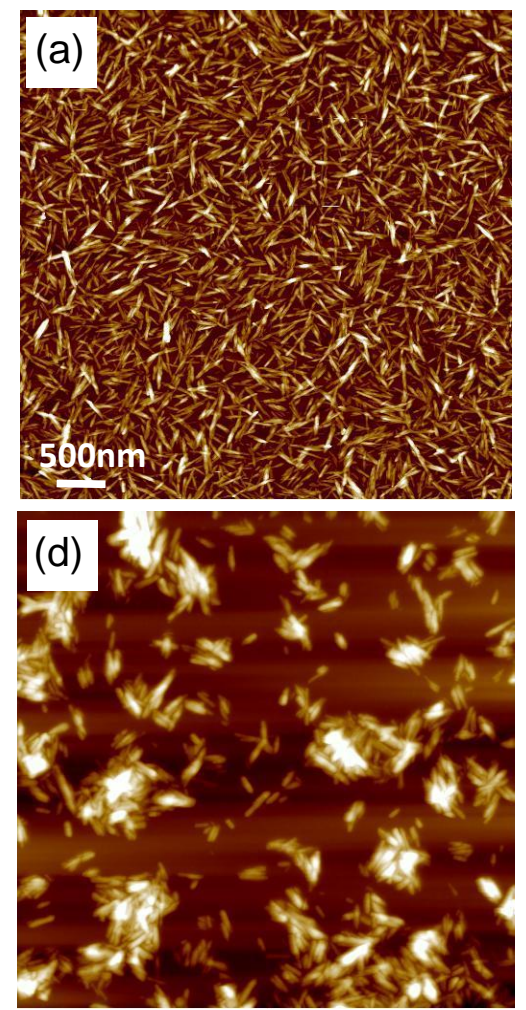
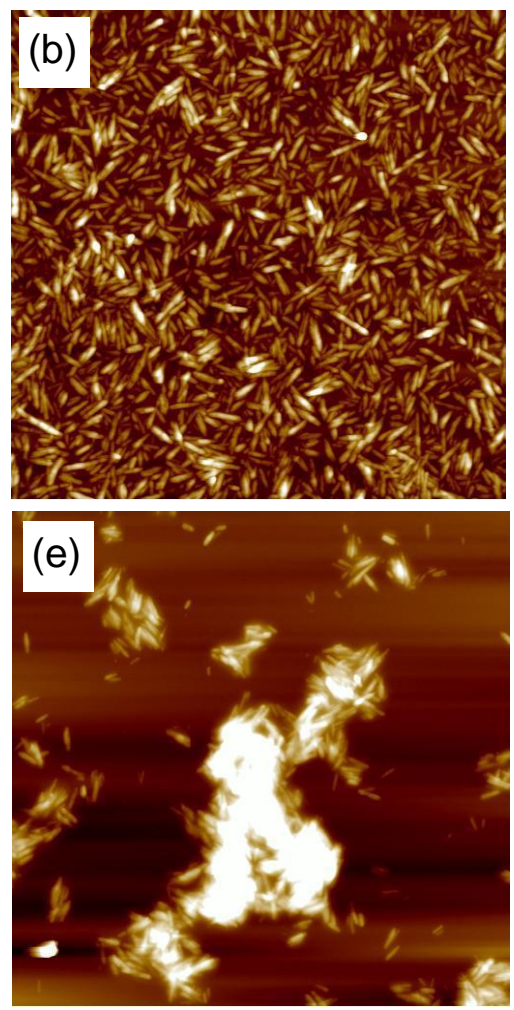
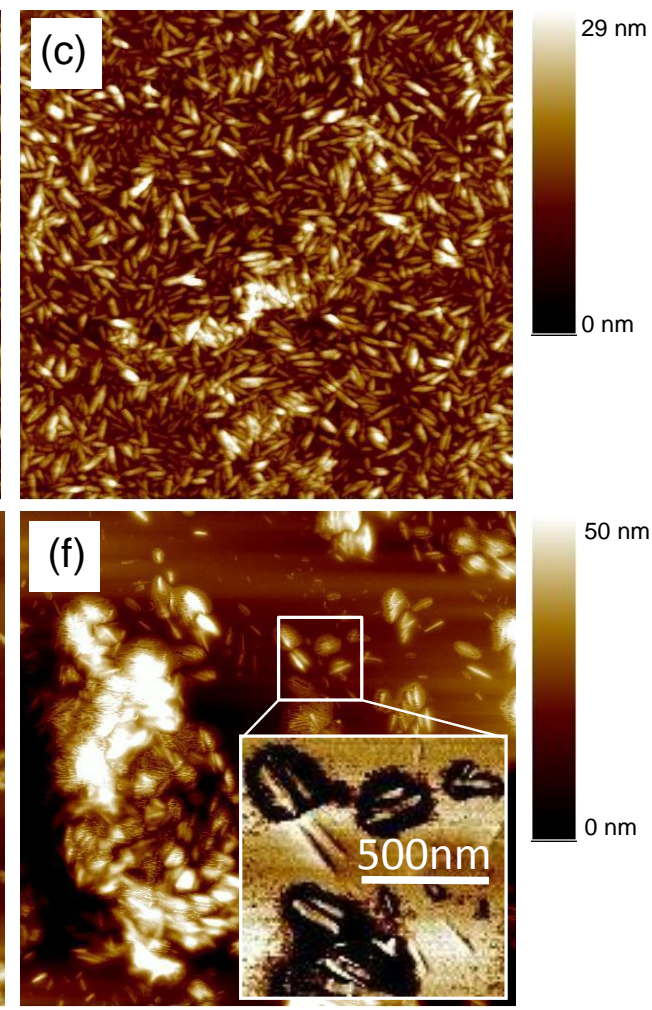

Figure 1. AFM height images $(5 \times 5 \mu \mathrm{m})$ of spin-coated films of unmodified CNCs (a) and poly(NiPAAm)-g-CNCs: LD (b), MD/LDP (c), VHD (d), MDP (e), and HDP (f). The films were air-dried before imaging. The inset in Figure $1 \mathrm{f}$ corresponds to an AFM phase image $(1 \times 1 \mu \mathrm{m})$ of the area indicated.

The thermo-responsiveness of unmodified CNCs and poly(NiPAAm)-g-CNCs in aqueous dispersions was analyzed by light transmittance. Although the same concentration was used for each dispersion, differences in the baseline $\%$ transmittance $\left(20{ }^{\circ} \mathrm{C}\right)$ were noted due to pre-aggregation of the grafted CNCs. Figure 2(a) shows normalized \% light transmittance versus temperature for 
unmodified CNCs and for six poly(NiPAAm)-g-CNCs samples. Unmodified CNCs showed no change in light transmittance in the range of 20 to $50{ }^{\circ} \mathrm{C}$; clearly, colloidal dispersions of CNCs were not responsive to temperature. With the presence of poly(NiPAAm) brushes grafted from CNCs, the dispersions (with varying graft density and degree of polymerization) showed a large shift in light transmittance; the onset of such changes was close to the LCST of poly(NiPAAm) of $32{ }^{\circ} \mathrm{C}$. This was taken as an indication of the thermo-responsiveness of the grafted CNCs which correlated well with the graft molecular weights. ${ }^{42}$ In earlier work by Okahata et al. it has been shown that poly(NiPAAm) maintains its thermo-responsive behavior when tethered to a substrate. ${ }^{28}$

Furthermore, the flocculation behavior of CNCs in aqueous dispersions, as determined by light transmittance, depended highly on the density and size of poly(NiPAAm) grafts. Such observation can be ascribed to the surface interactions between dispersed CNCs. For example, LD poly(NiPAAm)-g-CNCs containing the smallest amount of grafted polymer showed thermalresponse and the smallest aggregates above the LCST. Larger flocs were observed above the LCST as the graft density and/or degree of polymerization was increased, which indicated significant changes in the respective surface forces. With increased grafted amounts of polymer on CNCs, the electrostatic stabilization of the CNC dispersion was hindered. Light scattering did not resolve the forces that induced the more pronounced aggregations above the LCST of poly(NiPAAm) brushes; this will be discussed later in the section on surface interaction forces.

As previously shown, the LCST of free poly(NiPAAm) depends highly on the ionic strength of aqueous solutions due to the Hofmeister effect on dehydration of polymer chains. ${ }^{70}$ In our earlier reports, ${ }^{18}$ it has also been shown that poly(NiPAAm) chains tethered to a surface follow the Hofmeister effect. Figure 2(b) shows the LCST versus salt $[\mathrm{NaCl}]$ concentration of MD poly(NiPAAm)-g-CNCs, which decreased similarly as in the case of bulk polymer solutions. However, the decreasing trend in the LCST was more marked when compared to previous reports of the Hofmeister effect on poly(NiPAAm) chains. An LCST as low as ca. $23{ }^{\circ} \mathrm{C}$ was achieved in $1 \mathrm{M}$ $\mathrm{NaCl}$ aqueous solution, which was significantly lower than previous reports of free ${ }^{70}$ and tethered ${ }^{18}$ poly(NiPAAm) chains, with LCST at $c a .28^{\circ} \mathrm{C}$. All the remaining grafted CNCs samples showed a similar trend. 

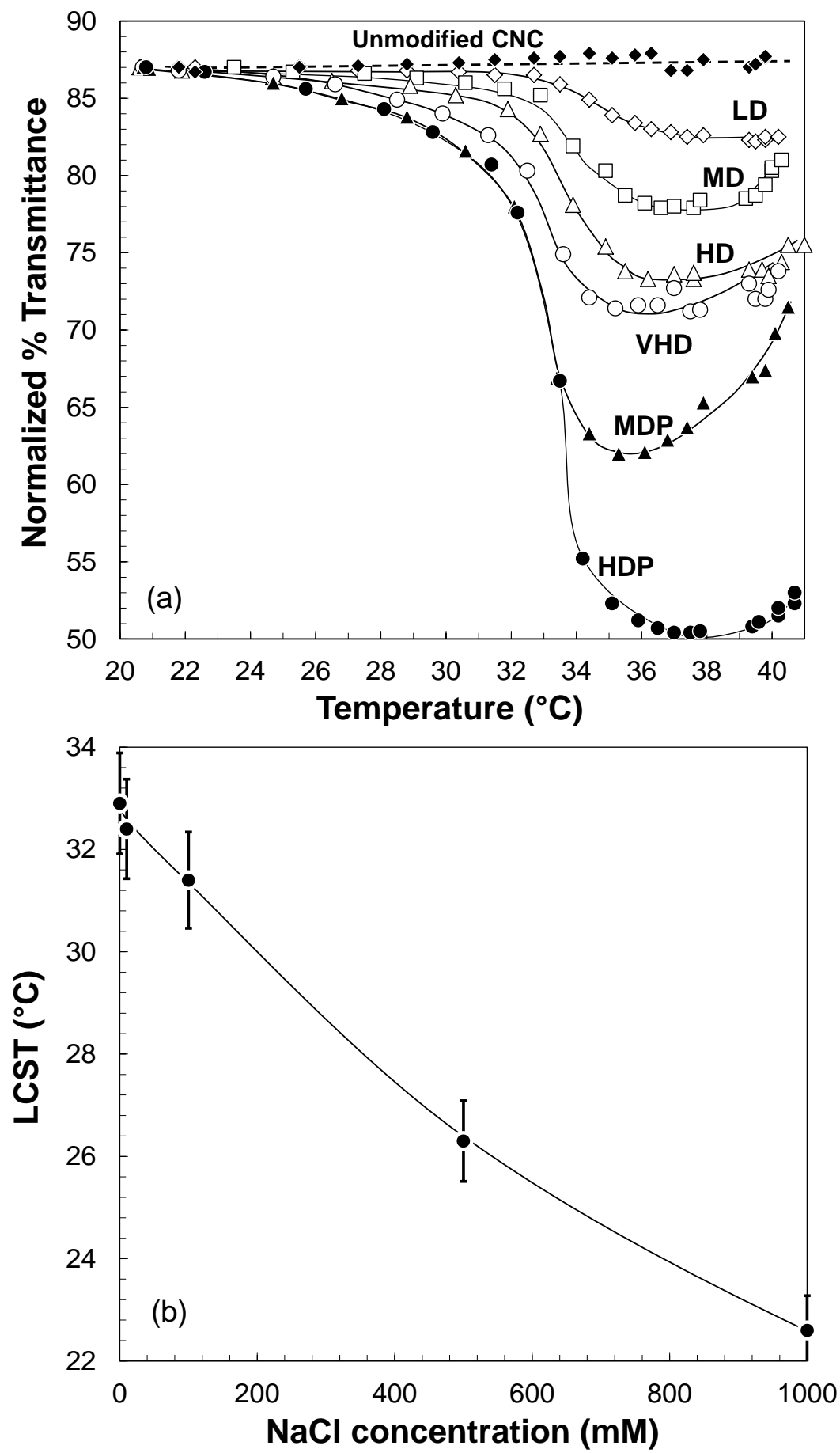

Figure 2. Normalized light transmittance versus temperature of aqueous dispersions of unmodified CNCs and poly(NiPAAm)-g-CNCs (LD, MD/LDP, HD, VHD, MDP, and HDP) (a). Figure (b) corresponds to the Lower Critical Solution Temperature of aqueous dispersions of MD poly(NiPAAm)-g-CNCs at various $\mathrm{NaCl}$ concentrations. The lines are added to guide the eye.

Viscosity measurements were performed to provide further evidence as to the aggregation and thermal responsiveness of CNCs. Aqueous dispersion of CNCs of a given concentration was 
equilibrated on a Peltier plate at $25{ }^{\circ} \mathrm{C}$ and the relative viscosity was monitored over a temperature ramp to $40{ }^{\circ} \mathrm{C}$ at a constant shear rate. The results of the rheological measurements are summarized in Figure 3. Typically, the relative viscosity of colloidal dispersions in a Newtonian fluid decreases as the temperature is raised, most simply explained by Arrhenius behavior of molecular kinetics. ${ }^{71}$ This was the case of dispersions of unmodified CNCs, as seen in Figure 3. On the other hand, the viscosity in aqueous solution of free poly(NiPAAm) was measured to confirm the LCST of $c a .32$ ${ }^{\circ} \mathrm{C} .{ }^{42}$ In stark contrast to the behavior of neat $\mathrm{CNCs}$, poly(NiPAAm)-g-CNCs showed significant increases in viscosity as the temperature approached the LCST of the grafted polymer brushes. Figure 3(a) indicated a response to temperature at $c a \cdot 30-32^{\circ} \mathrm{C}$ for various graft densities of poly(NiPAAm) brushes on CNCs. Below the LCST of grafted poly(NiPAAm) brushes, aqueous dispersions of poly(NiPAAm)-g-CNCs displayed viscosity values similar to that of the solvent (water). However as the LCST was reached, the dehydration of polymer brushes on the surfaces of $\mathrm{CNCs}$ created relatively more hydrophobic domains, and thus the onset of colloidal instability produced a $c a$. two-fold increase in apparent viscosity. Similarly in Figure 3(b), as the degree of polymerization of the grafts was varied, increased viscosity of dispersions was noted at $c a .30-32{ }^{\circ} \mathrm{C}$. Although it was unclear whether differences in LCST from that of free poly(NiPAAm) derived from differences in molecular weight or graft density, it was noted that with increased amounts of grafted poly(NiPAAm) (as determined previously by XPS) ${ }^{42}$ the thermo-responsive behavior was similar to that of free poly(NiPAAm). The observation that the viscosity of the poly(NiPAAm)-g-CNC dispersion increased as the LCST was approached is in agreement with the observations of Tam et al. who used viscosimetry to study conformational changes of free poly(NiPAAm). They indicated the possibility of aggregation and increase of the effective molecular weight of free poly(NiPAAm) in solution close to the LCST. ${ }^{72}$ The same authors reported that above the LCST phase separated polymer existed as colloidal particles which became unstable. This observation is also in agreement with our experimental results which indicated that colloidal instability occurred following phase separation of poly(NiPAAm)-g-CNCs. Such instabilities were more evident at temperatures above $34{ }^{\circ} \mathrm{C}$ (not reported in Figure 3). 

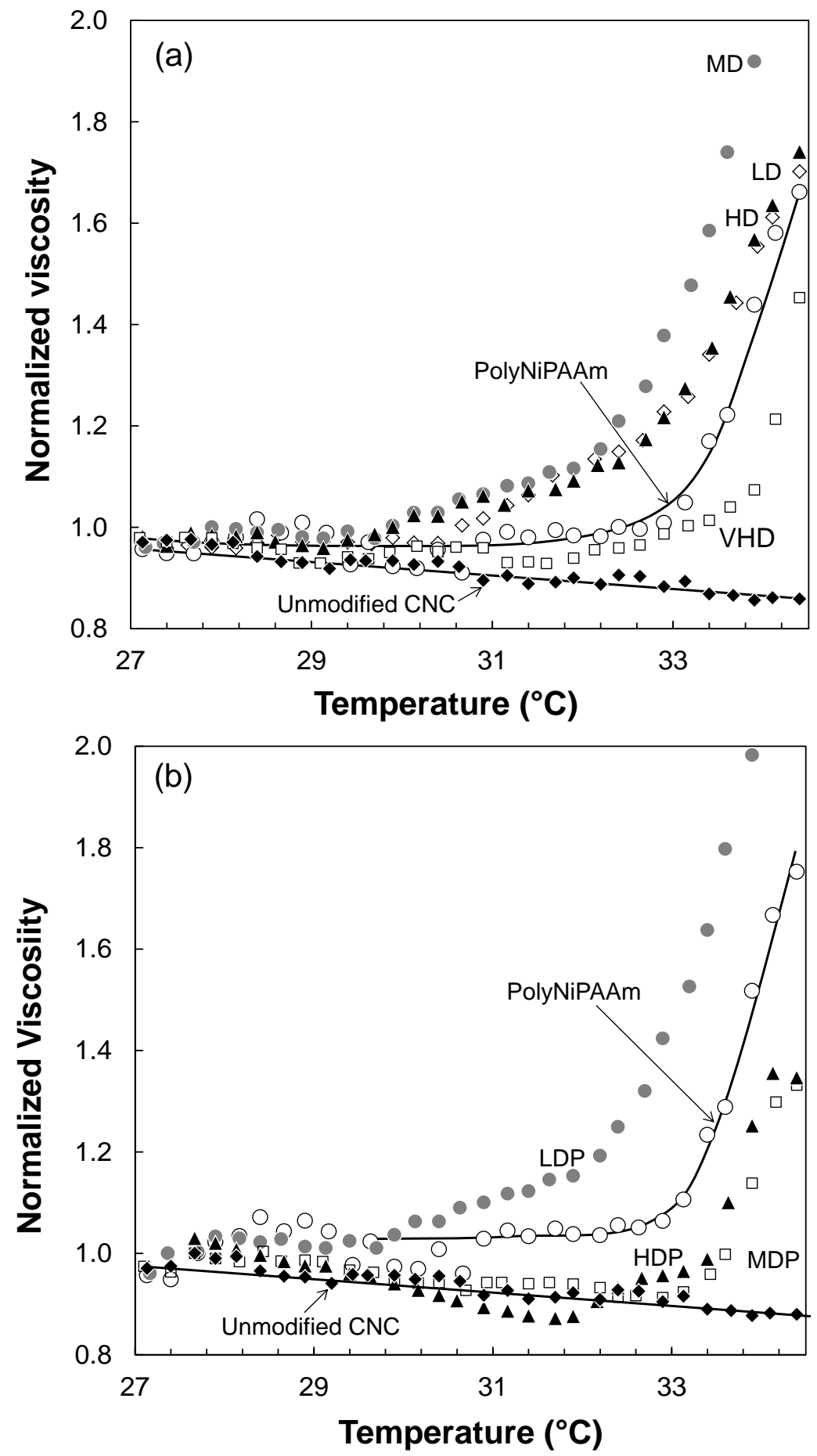

Figure 3. Viscosity of $\mathrm{CNC}$ dispersions at different temperatures normalized by the viscosity of the respective dispersions at $25{ }^{\circ} \mathrm{C}$ of poly(NiPAAm)-g-CNCs with various graft densities (a) and degrees of polymerization (b). The viscosity curves for dispersions of neat CNC and free poly(NiPAAM) solution are included as reference. The lines were added as guide to the eye.

As noted before, the responsiveness of poly(NiPAAm)-g-CNCs was not only affected by temperature, but by solution ionic strength. Although the results of light transmittance clearly 
showed the effect of $\mathrm{NaCl}$ on the LCST of grafted poly(NiPAAm) brushes (see Figure 2b), colloidal probe microscopy in aqueous media was further employed to shed light on the nature of the interfacial forces involving poly(NiPAAm) grafted from CNCs. Increasing the ionic strength of poly(NiPAAm)-g-CNCs dispersions not only decreased the Debye length of the electrostatic doublelayer derived from sulfate groups on $\mathrm{CNCs}$ surfaces, but also induced a partial collapse of poly(NiPAAm) brushes by means of the Hofmeister effect ${ }^{70}$ i.e., electrolytes disrupted the solvent's ability to fully dissolve polymer chains.

To further unveil the nature of the interactions, DLVO (electrostatic and van der Waals) and nonDLVO (steric, among others), the forces between a silica sphere and a surface coated with unmodified $\mathrm{CNCs}$ and poly(NiPAAm)-g-CNCs were probed by $\mathrm{CPM}$ at different ionic strengths. CPM could also be utilized to study the LCST behavior of poly(NiPAAm) brushes at various temperatures in aqueous media, however precise temperature control of the liquid cell chamber proved to be challenging considering the heat produced by the AFM laser. Spin coated films of unmodified $\mathrm{CNCs}$, LD, and $\mathrm{MD}$ poly(NiPAAm)-g-CNCs were uniform enough for force measurements (see Figure 1a-c). However, during attempts to measure the surface forces of LD poly(NiPAAm)-g-CNCs, it was evident that these films were unstable, most likely due to a low graft density of poly(NiPAAm) brushes on CNCs that could not remain adhered to the silicon wafer substrate after immersion in water. Since a stable, smooth and homogeneous topography is essential for successful force measurements, unmodified CNCs and the MD poly(NiPAAm)-g-CNCs were used (Figure 1b). Shown in Figure 4 are typical semi-log profiles of force versus separation of spincoated films of MD poly(NiPAAm)-g-CNCs and unmodified CNCs in $10 \mathrm{mM}[\mathrm{NaCl}]$. The onset of repulsive forces was observed at a relative surface separation of about 20-30 nm. The semi-log plot of normalized force versus separation between the surface coated with unmodified $\mathrm{CNC}$ and silica gave a linear relationship. This can be ascribed to osmotic repulsion between overlapping counterion clouds close to the negatively charged $\mathrm{CNC}$ and oxidized silica. The poly(NiPAAm)-g-CNCs on the other hand showed a non-linear trend. The repulsion was hypothesized in this case to be due to loss of conformational degree of freedom for the grafted polymer chains when the silica sphere approached. 


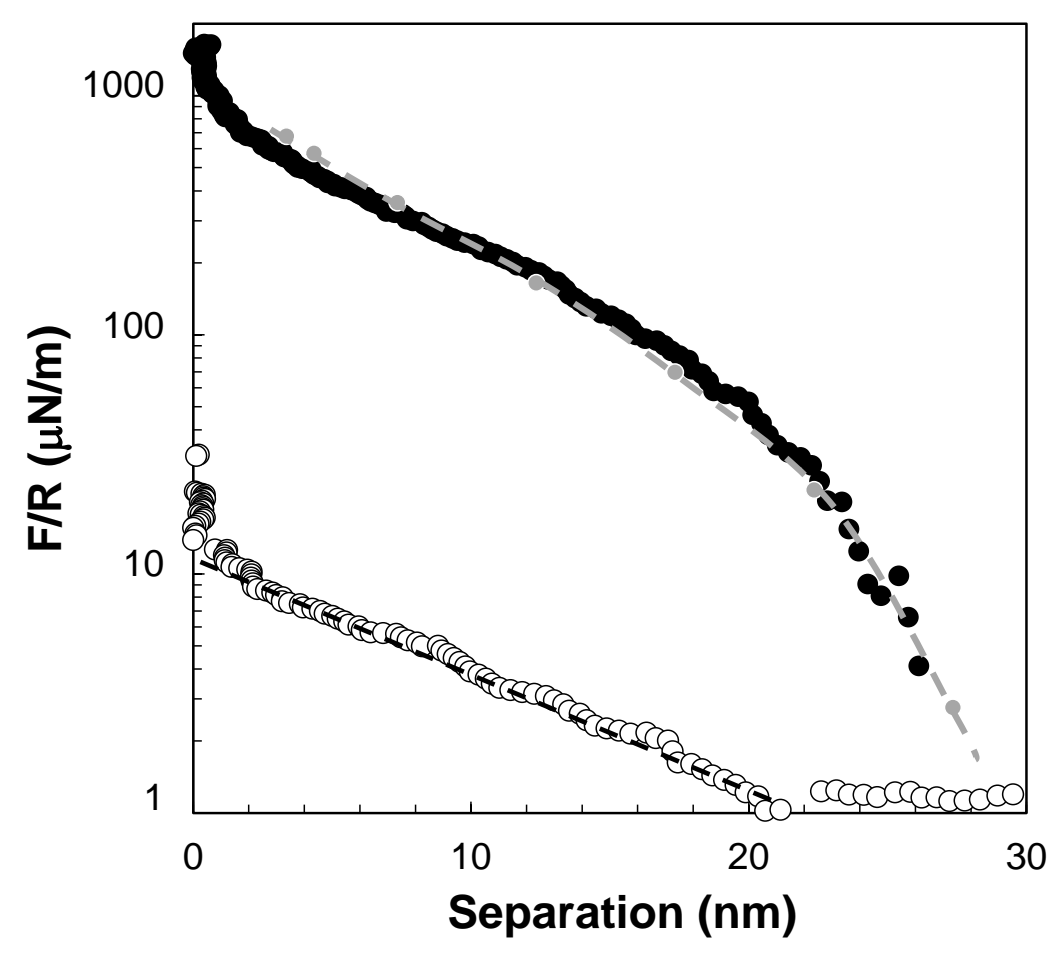

Figure 4. CPM interaction forces versus separation between a silica sphere against a flat layer spincoated on silica wafers consisting of unmodified CNCs (०) and MD poly(NiPAAm)-g-CNCs (•). The best fit to a model of steric interactions, Alexander-de Gennes, is shown in the case of MD poly(NiPAAm)-g-CNCs (see grey dash line) while a linear fit to indicate the expected slope for DLVO forces is included in the case of unmodified CNCs (see black dash line). The forces were measured in liquid medium, across aqueous $10 \mathrm{mM} \mathrm{NaCl}$ solution and normalized with the radius of the probing silica sphere.

Shown in Figure 5 are the normalized forces versus separation distance of an unmodified CNC film at various $[\mathrm{NaCl}]$ concentrations $\left(10,100\right.$, and $250 \mathrm{mM}$ with corresponding Debye lengths ${ }^{3}$ of $3.0,0.96$, and $0.61 \mathrm{~nm}$ at $25{ }^{\circ} \mathrm{C}$, respectively). As salt concentration was increased, the decay length of the repulsion decreased, supporting the assumption that the forces are in this case of electrostatic origin. The grafting of poly(NiPAAm) brushes from CNCs changed the interfacial interactions of the silica sphere with the poly(NiPAAm)-g-CNC coated surface. As shown in Figure 6, a semi-log plot showed clearly repulsion derived from steric forces and the effect of increased ionic strength. At low 
salt concentration $(10 \mathrm{mM}[\mathrm{NaCl}])$, poly(NiPAAm) brushes were in a highly extended state in solution, which gave steric repulsion starting at ca. $25 \mathrm{~nm}$ relative separation. According to the average molecular weight of the MD grafted poly(NiPAAm) chains, ${ }^{42}$ the length of a fully-stretched polymer chain was calculated to be $\sim 14.5 \mathrm{~nm}$, however poly(NiPAAm) brushes were expected to be in a coiled confirmation, thus one could suspect a smaller brush layer thickness. Additionally, poly(NiPAAm) brushes were expected to be coated over the entire surface of CNCs, thus a partially swollen polymer layer may have existed adsorbed to the silica substrate. The observed onset of steric repulsion at $\sim 25 \mathrm{~nm}$ may have been an indication that a partially swollen polymer layer existed underneath CNCs while adhered to the silica surface in addition to extended polymer brushes free for interaction with the silica probe. As the concentration of salt was increased ten-fold, steric forces were not apparent until ca. $18 \mathrm{~nm}$ inter-surface separation distance, which indicated a partial collapse of grafted poly(NiPAAm) brushes and the adsorbed polymer layer (see Figure 7).

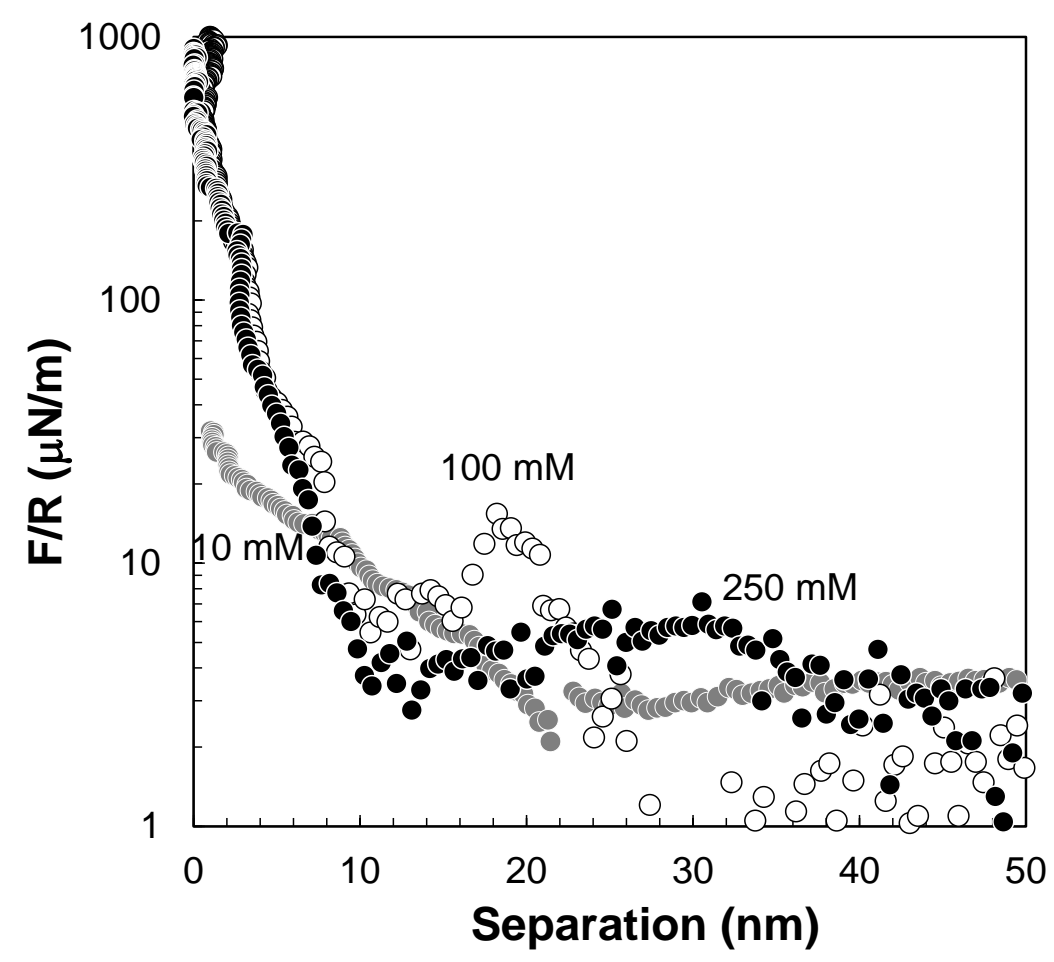

Figure 5. CPM interaction forces normalized with the radius of the silica sphere probe as a function of separation distance against a flat film of unmodified CNCs. The forces were measured in aqueous $\mathrm{NaCl}$ solutions of various concentrations, $10 \mathrm{mM}(\bullet), 100 \mathrm{mM}(\circ)$ and $250 \mathrm{mM}(\bullet)$. 


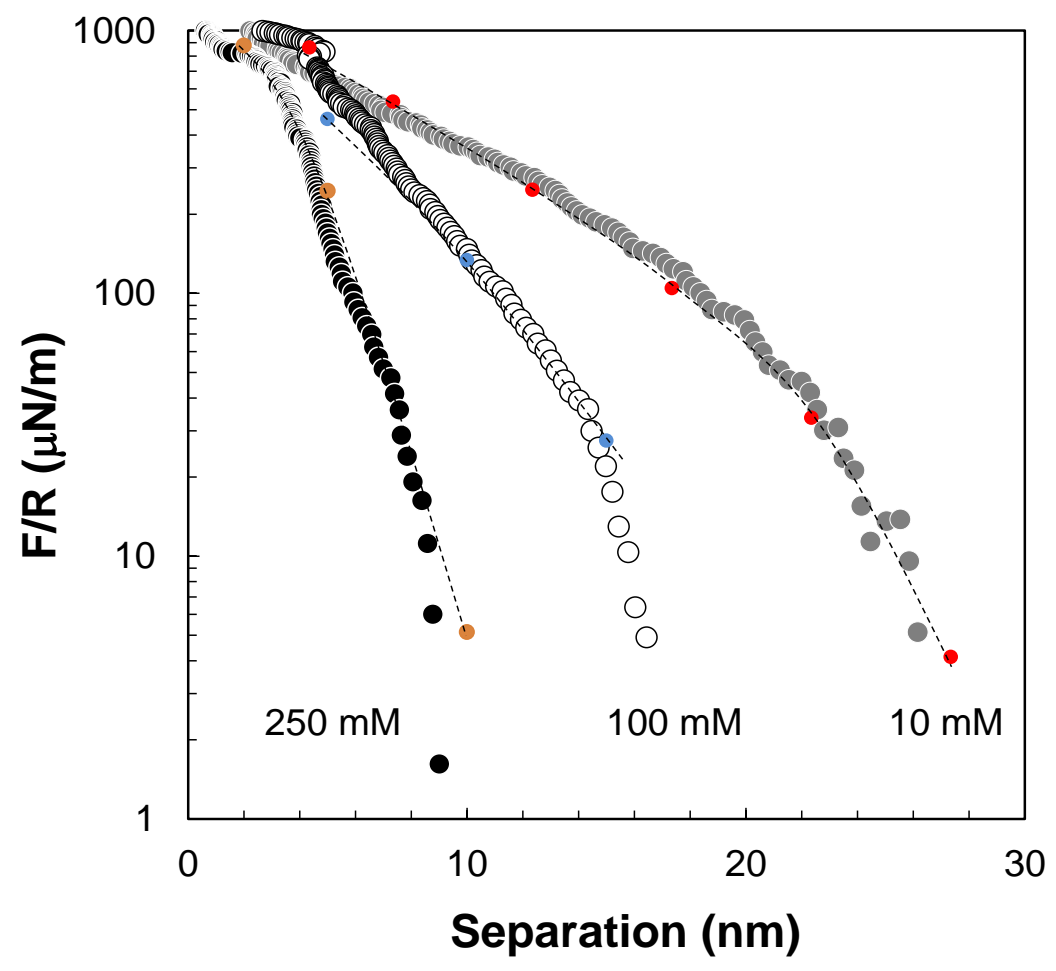

Figure 6. CPM interaction forces between a silica sphere probe and flat films of $\mathrm{MD}$ poly(NiPAAm)-g-CNCs as a function of the separation distance. The forces were measured in aqueous $\mathrm{NaCl}$ solutions of various concentrations (10 mM (•), $100 \mathrm{mM}(\circ)$ and $250 \mathrm{mM}(\bullet))$ and were normalized with the radius of the silica sphere. The corresponding best fits to the Alexander-de Gennes equation are added as a reference (see respective dash lines).

At $250 \mathrm{mM}$, steric repulsion was not evident until separations of $c a .10 \mathrm{~nm}$, revealing increased collapse of the grafted polymer chains. Overall, the steric forces were only evident at $c a .30 \mathrm{~nm}$ and smaller separation distances, which were relatively short when compared to reports of similar systems. ${ }^{34}$ However, this range for steric interaction forces is consistent with the fact that an asymmetric system was dealt with (brushes only present on one of the surfaces) and also the small molecular weight of the polymer grafts used $(\sim 10 \mathrm{kDa})$.

In the past, Alexander-de Gennes theory was developed strictly for tethered polymers at high graft densities, ${ }^{35,36}$ but it has been shown to be applicable also to adsorbed polymer layers. ${ }^{73}$ The graft densities in poly(NiPAAm)-g-CNCs were expected to be relatively low, given that the polymers were tethered to CNCs. However, in a study performed by Notley ${ }^{34}$ on the conformation of adsorbed 
poly(NiPAAm) layers on silica in a binary solvent mixture, steric forces were apparent, and it was determined that the Alexander-de Gennes model could be used as a reasonable fit at intermediate to large surface separations, even with low graft densities. As such, an Alexander-de Gennes model was fit to the experimental curves at each salt concentration in order to provide further evidence of forces of steric origin. Except for large separation distances, the model fit reasonably well with the force profiles. However, this observation was taken as qualitative evidence and no attempt was made to interpret the nature of such steric effects and the ensuing components (elastic, osmotic and others).

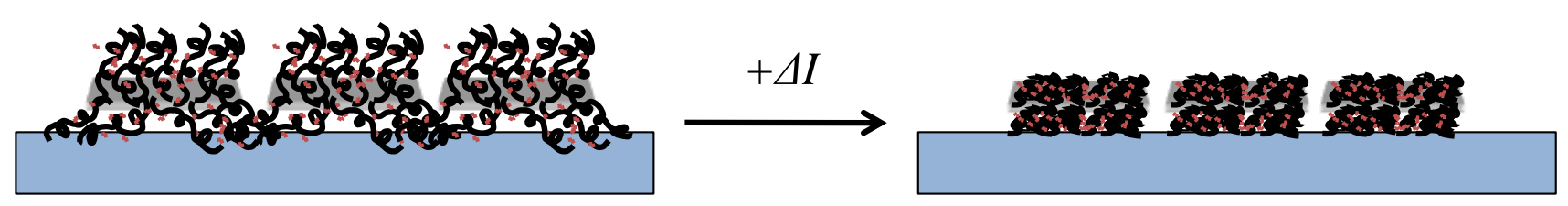

Figure 7. Schematic illustration of poly(NiPAAm)-g-CNCs adsorbed on silica substrates subjected to increased ionic strength in aqueous media.

Shown in Figure 8(a-b) are linear force versus separation distance profiles of a silica colloidal sphere approaching (IN) and retracting from (OUT) a spin-coated film of MD poly(NiPAAm)-g$\mathrm{CNCs}$ in $10 \mathrm{mM}$ (a) and $100 \mathrm{mM}(\mathrm{b})[\mathrm{NaCl}]$ aqueous solution. Some initial attraction was observed upon approaching extended poly(NiPAAm) brushes, most likely due to bridging attraction between the poly(NiPAAM) brushes containing secondary amine moieties and the anionic silica sphere in aqueous medium. As mentioned earlier, poly(NiPAAm)-g-CNCs readily adsorbed to oxidized silicon wafers without the use of anchoring polymer, thus an attraction between poly(NiPAAm) brushes and the silica colloidal probe was expected. Hydrogen bonding between the amide functionalities of poly(NiPAAm) has been the proposed mechanism of adsorption to silica surfaces below the LCST. ${ }^{34,74}$ Attraction was detected at separations of $c a .60 \mathrm{~nm}$ at low electrolyte concentration. A long range attractive force was also noted previously for the case of adsorbed poly(NiPAAm) layers in a partially collapsed state. ${ }^{34}$ At $c a .30 \mathrm{~nm}$ separation, repulsion between the silica sphere and the grafted $\mathrm{CNCs}$ was observed. Significant adhesion between the silica sphere and grafted $\mathrm{CNCs}$ was evident at $10 \mathrm{mM}[\mathrm{NaCl}]$, seen in Figure $8(\mathrm{a})$, most likely due to strong 
interactions between poly(NiPAAm) brushes and the silica surface. Increasing the electrolyte concentration to $100 \mathrm{mM}$ decreased the adhesion between the silica surface and grafted $\mathrm{CNCs}$ and no attraction on approach was noted (Figure 8b). This was explained by the partial collapse of poly(NiPAAm) brushes. From the retraction force curves, pull-off forces were calculated using the SPIP code (see Table 1). Overall, as the salt concentration was increased, a partial collapse of poly(NiPAAm) brushes and reduced adhesion took place. Partial collapse of poly(NiPAAm) brushes was believed to have decreased non-electrostatic polymer bridges formed between the silica sphere and spin-coated surface. Further, this finding could have been due to ion adsorption at hydrogen bonding sites on NiPAAm monomers leading to decreased adsorption of poly(NiPAAm) brushes to the silica probe as has been described in similar systems. ${ }^{75,76}$ In addition, Meagher et al. ${ }^{77}$ noted unexpected attractive interactions between silica and low graft density poly(ethylene oxide) (PEO) brushes which was hypothesized to originate from the adsorption of PEO chains onto the silica probe during approach and that increased adsorption occurred with decreased separation distance. In addition, a small fraction of longer PEO chains within the polydisperse brushes could have given rise to force interactions at farther than expected separations. In another study, Goodman et al. ${ }^{78}$ found that low graft density polymer brushes gave rise to attractive forces that became repulsive upon increased graft density, which was explained by a decreased mobility of the polymer brushes within the layer. In the present case, poly(NiPAAm) brushes had a large polydispersity index ${ }^{42}$ which could be the reason for the longer range interactions reported. As the ionic strength was increased, poly(NiPAAm) brush collapse was evident which also decreased polymer brush mobility. This effect could explain the decreased attractive forces upon approach of the silica probe and thus, the decreased pull-off force observed with increased ionic strength. In the cases of unmodified CNCs films, a minimal adhesion was observed with pull-off forces typically less than $1 \mathrm{nN}$ (see Table 1). 

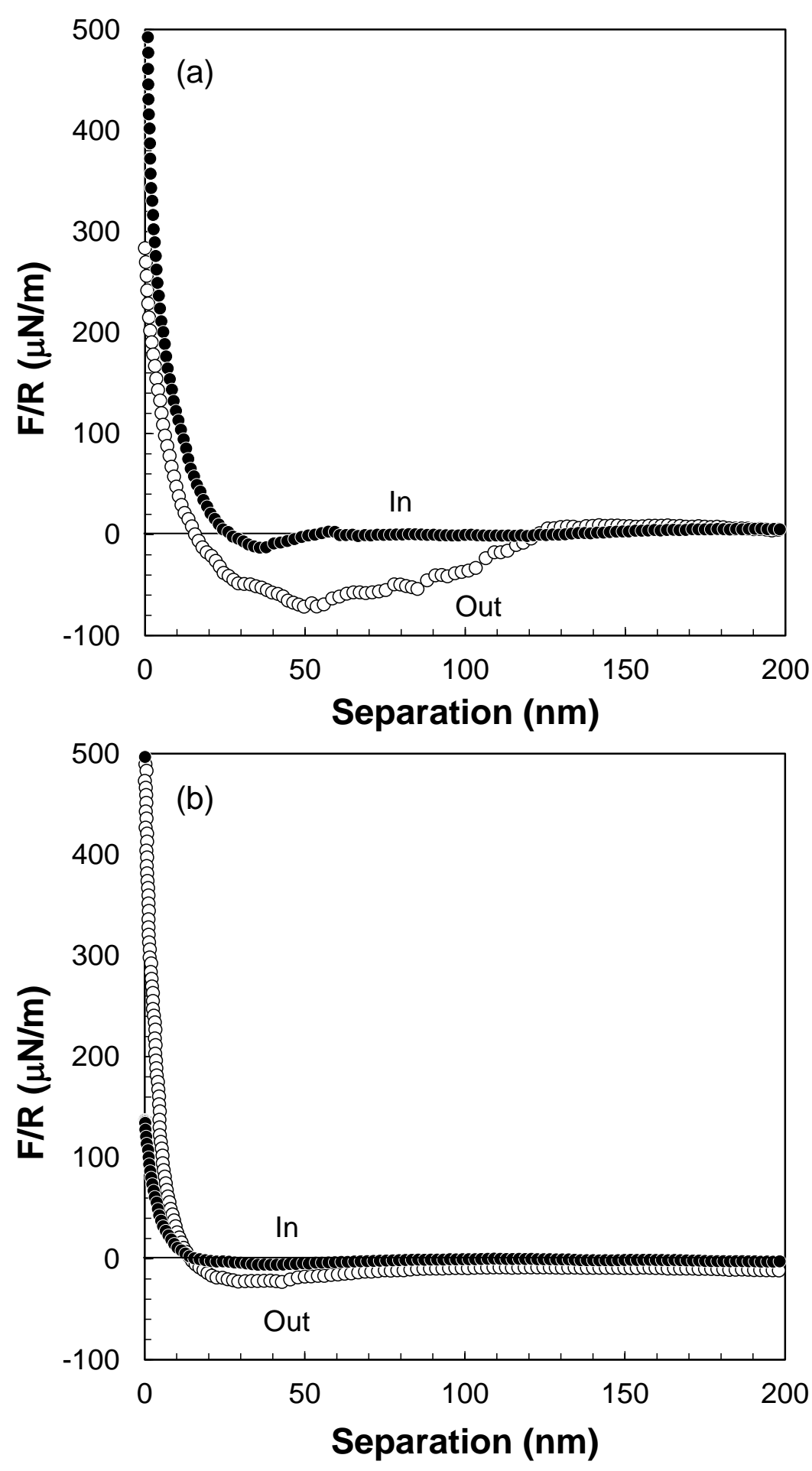

Figure 8. CPM normalized interaction force versus separation distance for a silica sphere probe against a flat film of MD poly(NiPAAm)-g-CNCs measured in $\mathrm{NaCl}$ aqueous solutions of $10 \mathrm{mM}$ (a), and $100 \mathrm{mM}$ (b) concentrations. The force profiles recorded upon approach (In, •), and on separation (Out, ○) are included in each case. 
Table 1. Pull-off (adhesion) forces of unmodified CNCs and MD poly(NiPAAm)-g-CNCs against silica in aqueous solution of various salt concentrations.

\begin{tabular}{lll}
\hline$[\mathrm{NaCl}]$ & Adhesion $(\mathrm{nN})$, & Adhesion $(\mathrm{nN}), \mathrm{MD}$ \\
& unmodified CNCs & poly(NiPAAm)-g-CNCs \\
\hline $10 \mathrm{mM}$ & $-0.4 \pm 0.3$ & $-9.9 \pm 1.0$ \\
\hline $100 \mathrm{mM}$ & $-0.7 \pm 0.3$ & $-5.8 \pm 1.0$ \\
& & \\
\hline $250 \mathrm{mM}$ & $-0.6 \pm 0.4$ & $-5.5 \pm 0.6$
\end{tabular}

Cellulose nanocrystals are expected to be interesting substrates for functional surface modifications, especially due to their low cost and properties as compared to other types of nanoparticles. By means of stimuli-responsive polymer grafting, their self-assembly behavior can be controlled, as shown in the preceding discussion. In general, higher grafting densities and longer polymer brushes yielded larger aggregations or flocs after the LCST of poly(NiPAAm) was reached. We have demonstrated that ultrathin films of grafted CNCs give rise to steric repulsion forces that responded similarly to changes in ionic strength as observed in experiments with aqueous dispersions of grafted CNCs. Colloidal probe microscopy allowed better understanding of the origins of attractive and repulsion forces in these systems. The onset of steric repulsion correlated with the theoretical length of the free polymer brushes grafted from cellulose nanocrystals and a partially swollen polymer layer adsorbed to the silica substrate. Thus, by varying the grafting density and/or molecular weight of stimuli-responsive polymer brushes grafted from CNCs, their surface interaction forces in dispersion and as a surface coating could be controlled by temperature and/or ionic strength. In this way, phenomena such as rheological behavior, adhesion, lubrication, and drugrelease properties could be tailored.

\section{Conclusions}

Poly(NiPAAm) brushes grafted from CNCs were observed under AFM, especially in the case of chains with the highest degree of polymerization, ca. $40 \mathrm{kDa}$. Differences in surface aggregation in spin-coated films of poly(NiPAAm)-g-CNCs were also observed and related to the graft density and 


\section{Acknowledgments}

The authors would like to acknowledge Paula Eronen and Karoliina Junka (Aalto University) for preparation of colloidal probes. This project was supported by the National Research Initiative of the USDA Cooperative State Research, Education and Extension Service, grant number 2007-3550418290. Also, the USDA NNF program grant number 2007-38420-17772 and TEKES's LignoCell (Finland) are gratefully acknowledged. 


\section{References}

(1) Tadros, T., Adv. Colloid Interface Sci. 2003, 104, 191-226.

(2) Napper, D. H., Polymeric Stabilization of Colloidal Dispersions, Academic Press: London, 1985.

(3) Israelachvili, J. N., Intermolecular and Surface Forces, Academic Press: London, 1985.

(4) Fleer, G. J.; Cohen Stuart, M. A.; Scheutjens, J. M. H. M.; Cosgrove, T.; Vincent, B., Polymers at Interfaces, Chapman \& Hall: London, 1993.

(5) Whitesides, G. M.; Ostuni, E.; Takayama, S.; Jiang, X.; Ingber, D. E., Annu. Rev. Biomed. Eng. 2001, 3, 335-373.

(6) Zhang, S.; Yan, L.; Altman, M.; Lassle, M.; Nugent, H.; Frankel, F.; Lauffenburger, D. A.; Whitesides, G. M.; Rich, A., Biomaterials 1999, 20, 1213-1220.

(7) Blawas, A. S.; Reichert, W. M., Biomaterials 1998, 19, 595-609.

(8) Ratner, B. D.; Bryant, S. J., Annu. Rev. Biomed. Eng. 2004, 6, 41-75.

(9) Mano, J. F., Adv. Eng. Mater. 2008, 10, 515-527.

(10) Specht, E. H.; Neuman, A.; Neher, H. T. Acrylamides. U. S. pat. 2,773,063 (Dec. 4, 1956).

(11) Schild, H. G., Prog. Polym. Sci. 1992, 17, 163-249.

(12) Heskins, M.; Guillet, J. E., J. Macromol. Sci., Chem. 1968, 2, 1441-55.

(13) Matsuyama, A.; Tanaka, F., Phys. Rev. Lett. 1990, 65, 341-4.

(14) Mauricio, M. R.; Carvalho, G. M.; Radovanovic, E.; Muniz, E. C.; Rubira, A. F., Mater. Sci. Eng., C 2009, 29, 594-598.

(15) Wang, W.; Chen, L., J. Appl. Polym. Sci. 2007, 104, 1482-1486.

(16) Yin, J.; Ge, Z.; Liu, H.; Liu, S., J. Polym. Sci., Part A. 2009, 47, 2608-2619.

(17) Yamada, N.; Okano, T.; Sakai, H.; Karikusa, F.; Sawasaki, Y.; Sakurai, Y., Makromol. Chem., Rapid Commun. 1990, 11, 571-6.

(18) Jhon, Y. K.; Bhat, R. R.; Jeong, C.; Rojas, O. J.; Szleifer, I.; Genzer, J., Macromol. Rapid Commun. 2006, 27, 697-701. 
(19) Lindqvist, J.; Nystroem, D.; Oestmark, E.; Antoni, P.; Carlmark, A.; Johansson, M.; Hult, A.; Malmström, E., Biomacromolecules 2008, 9, 2139-2145.

(20) Cole, M. A.; Voelcker, N. H.; Thissen, H.; Horn, R. G.; Griesser, H. J., Soft Matter 2010, 6, 2657-2667.

(21) Goodman, D.; Kizhakkedathu, J. N.; Brooks, D. E., Langmuir 2004, 20, 3297-3303.

(22) Ishida, N.; Kobayashi, M., J. Colloid Interface Sci. 2006, 297, 513-519.

(23) Jones, D. M.; Smith, J. R.; Huck, W. T. S.; Alexander, C., Adv. Mater. 2002, 14, 1130-1134.

(24) He, Q.; Kuller, A.; Grunze, M.; Li, J., Langmuir 2007, 23, 3981-7.

(25) Liu, G.; Cheng, H.; Yan, L.; Zhang, G., J. Phys. Chem. B 2005, 109, 22603-22607.

(26) Hernandez-Guerrero, M.; Min, E.; Barner-Kowollik, C.; Mueller, A. H. E.; Stenzel, M. H., J. Mater. Chem. 2008, 18, 4718-4730.

(27) Ishida, N.; Biggs, S., Macromolecules 2010, 43, 7269-7276.

(28) Okahata, Y.; Noguchi, H.; Seki, T., Macromolecules 1986, 19, 493-4.

(29) Cole, M. A.; Voelcker, N. H.; Thissen, H.; Griesser, H. J., Biomaterials 2009, 30, 1827-1850.

(30) Akiyama, Y.; Kushida, A.; Yamato, M.; Kikuchi, A.; Okano, T., J. Nanosci. Nanotechnol. 2007, 7, 796-802.

(31) Ishida, N.; Biggs, S., Langmuir 2007, 23, 11083-11088.

(32) Mendez, S.; Andrzejewski, B. P.; Canavan, H. E.; Keller, D. J.; McCoy, J. D.; Lopez, G. P.; Curro, J. G., Langmuir 2009, 25, 10624-10632.

(33) Kidoaki, S.; Ohya, S.; Nakayama, Y.; Matsuda, T., Langmuir 2001, 17, 2402-2407.

(34) Notley, S. M., J. Phys. Chem. B 2008, 112, 12650-12655.

(35) Alexander, S., J. Phys. (Paris) 1977, 38, 983-7.

(36) De Gennes, P. G., Macromolecules 1980, 13, 1069-75.

(37) Milner, S. T.; Witten, T. A.; Cates, M. E., Macromolecules 1988, 21, 2610-19.

(38) Hamad, W., Can. J. Chem. Eng. 2006, 84, 513-519.

(39) Kamel, S., eXPRESS Polym. Lett. 2007, 1, 546-575.

(40) Yi, J.; Xu, Q.; Zhang, X.; Zhang, H., Cellulose 2009, 16, 989-997.

(41) Filpponen, I.; Argyropoulos, D. S., Biomacromolecules 2010, 11, 1060-1066. 
(42) Zoppe, J. O.; Habibi, Y.; Rojas, O. J.; Venditti, R. A.; Johansson, L.-S.; Efimenko, K.;

Osterberg, M.; Laine, J., Biomacromolecules 2010, 11, 2683-2691.

(43) Utsel, S.; Malmstroem, E. E.; Carlmark, A.; Wågberg, L., Soft Matter 2010, 6, 342-352.

(44) Dufresne, A., Can. J. Chem. 2008, 86, 484-494.

(45) Habibi, Y.; Lucia, L. A.; Rojas, O. J., Chem. Rev. 2010, 110, 3479-3500.

(46) Holmberg, M.; Wigren, R.; Erlandsson, R.; Claesson, P. M., Colloids Surf., A 1997, 129,130, 175-183.

(47) Zauscher, S.; Klingenberg, D. J., Nordic Pulp Pap. Res. J. 2000, 15, 459-468.

(48) Zauscher, S.; Klingenberg, D. J., J. Colloid Interface Sci. 2000, 229, 497-510.

(49) Notley, S. M.; Eriksson, M.; Wagberg, L.; Beck, S.; Gray, D. G., Langmuir 2006, 22, 31543160.

(50) Stiernstedt, J.; Nordgren, N.; Wagberg, L.; Brumer, H.; Gray, D. G.; Rutland, M. W., J. Colloid Interface Sci. 2006, 303, 117-123.

(51) Salmi, J. PhD Thesis, Helsinki University of Technology, Espoo, 2009.

(52) Carambassis, A.; Rutland, M. W., Langmuir 1999, 15, 5584-5590.

(53) Rutland, M. W.; Carambassis, A.; Willing, G. A.; Neuman, R. D., Colloids Surf., A 1997, 123-124, 369-374.

(54) Notley Shannon, M.; Wagberg, L., Biomacromolecules 2005, 6, 1586-91.

(55) Notley, S. M.; Chen, W.; Pelton, R., Langmuir 2009, 25, 6898-6904.

(56) Notley, S. M.; Pettersson, B.; Wagberg, L., J. Am. Chem. Soc. 2004, 126, 13930-13931.

(57) Nigmatullin, R.; Lovitt, R.; Wright, C.; Linder, M.; Nakari-Setala, T.; Gama, M., Colloids Surf B 2004, 35, 125-35.

(58) Guan, Y.; Xiao, H.; Sullivan, H.; Zheng, A., Carbohydr. Polym. 2007, 69, 688-696.

(59) Leporatti, S.; Sczech, R.; Riegler, H.; Bruzzano, S.; Storsberg, J.; Loth, F.; Jaeger, W.; Laschewsky, A.; Eichhorn, S.; Donath, E., J. Colloid Interface Sci. 2005, 281, 101-111.

(60) Paananen, A.; Osterberg, M.; Rutland, M.; Tammelin, T.; Saarinen, T.; Tappura, K.; Stenius, P., ACS Symp. Ser. 2004, 864, 269-290.

(61) Radtchenko, I. L.; Papastavrou, G.; Borkovec, M., Biomacromolecules 2005, 6, 3057-3066. 
(62) Cranston, E. D.; Gray, D. G.; Rutland, M. W., Langmuir 2010, 26, 17190-17197.

(63) Neuman, R. D.; Berg, J. M.; Claesson, P. M., Nord. Pulp Pap. Res. J. 1993, 8, 96-104.

(64) Holmberg, M.; Berg, J.; Stemme, S.; Oedberg, L.; Rasmusson, J.; Claesson, P., J. Colloid Interface Sci. 1997, 186, 369-381.

(65) Osterberg, M., J. Colloid Interface Sci. 2000, 229, 620-627.

(66) Lefebvre, J.; Gray, D. G., Cellulose 2005, 12, 127-134.

(67) Hutter, J. L.; Bechhoefer, J., Rev. Sci. Instrum. 1993, 64, 1868-73.

(68) Ducker, W. A.; Senden, T. J.; Pashley, R. M., Nature 1991, 353, 239-41.

(69) Ducker, W. A.; Senden, T. J.; Pashley, R. M., Langmuir 1992, 8, 1831-6.

(70) Schild, H. G.; Tirrell, D. A., J. Phys. Chem. 1990, 94, 4352-6.

(71) Atkins, P.; De Paula, J., Physical Chemistry, 9th ed.; W. H. Freeman and Company: New York, 2010.

(72) Tam, K. C.; Wu, X. Y.; Pelton, R. H., Polymer 1992, 33, 436-8.

(73) De Gennes, P. G., Adv. Colloid Interface Sci. 1987, 27, 189-209.

(74) Tanahashi, T.; Kawaguchi, M.; Honda, T.; Takahashi, A., Macromolecules 1994, 27, 606-7.

(75) Schneider, J.; Berndt, P.; Haverstick, K.; Kumar, S.; Chiruvolu, S.; Tirrell, M., Langmuir 2002, 18, 3923-3931.

(76) Zhu, X.; DeGraaf, J.; Winnik, F. M.; Leckband, D., Langmuir 2004, 20, 10648-10656.

(77) Hamilton-Brown, P.; Gengenbach, T.; Griesser, H. J.; Meagher, L., Langmuir 2009, 25, 9149-9156.

(78) Goodman, D.; Kizhakkedathu, J. N.; Brooks, D. E., Langmuir 2004, 20, 2333-2340. 
Table of Contents (TOC) graphic $(3.6 \times 8.9 \mathrm{~cm})$ :

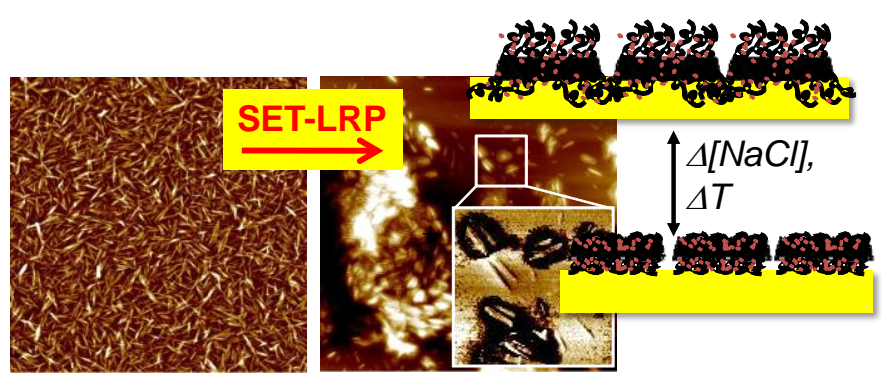

